\title{
Plasticity
}

Dev Neurosci 2015;37:440-452

DOI: $10.1159 / 000375487$
Received: August 18, 2014

Accepted after revision: January 26, 2015

Published online: March 17, 2015

\section{Dissociation in the Effects of Induced Neonatal Hypoxia-Ischemia on Rapid Auditory Processing and Spatial Working Memory in Male Rats}

\author{
Amanda L. Smith ${ }^{a}$ Michelle Alexander ${ }^{b}$ James J. Chrobak ${ }^{a}$ \\ Ted S. Rosenkrantz ${ }^{\mathrm{c}}$ R. Holly Fitch ${ }^{\mathrm{a}}$ \\ a Department of Psychology, Behavioral Neuroscience Division, University of Connecticut, Storrs, Conn., \\ ${ }^{b}$ Division of Neonatology, Department of Pediatrics, University of Minnesota, Minneapolis, Minn., and \\ ${ }^{c}$ Department of Pediatrics, University of Connecticut Health Center, Farmington, Conn., USA
}

\section{Key Words}

Rapid auditory processing · Spatial working memory . Hypoxia-ischemia $\cdot$ Correlation $\cdot$ Rodent model $\cdot$ Neural substrates · Neurobehavioral · Preterm infant

\begin{abstract}
Infants born prematurely are at risk for cardiovascular events causing hypoxia-ischemia ( $\mathrm{HI}$; reduced blood and oxygen to the brain). $\mathrm{HI}$ in turn can cause neuropathology, though patterns of damage are sometimes diffuse and often highly variable (with clinical heterogeneity further magnified by rapid development). As a result, though $\mathrm{HI}$ injury is associated with long-term behavioral and cognitive impairments in general, pathology indices for specific infants can provide only limited insight into individual prognosis. The current paper addresses this important clinical issue using a rat model that simulates unilateral $\mathrm{HI}$ in a late preterm infant coupled with long-term behavioral evaluation in two processing domains - auditory discrimination and spatial learning/memory. We examined the following: (1) whether deficits on one task would predict deficits on the other (suggesting that subjects with more severe injury perform worse across all cognitive domains) or (2) whether domain-specific outcomes among $\mathrm{HI}$-injured subjects would be uncorrelated
\end{abstract}

(suggesting differential damage to orthogonal neural systems). All animals (sham and $\mathrm{HI}$ ) received initial auditory testing and were assigned to additional auditory testing (group A) or spatial maze testing (group B). This allowed within-task (group A) and between-task (group B) correlation. Anatomic measures of cortical, hippocampal and ventricular volume (indexing $\mathrm{HI}$ damage) were also obtained and correlated against behavioral measures. Results showed that auditory discrimination in the juvenile period was not correlated with spatial working memory in adulthood (group B) in either sham or HI rats. Conversely, early auditory processing performance for group A HI animals significantly predicted auditory deficits in adulthood ( $p=0.05$; no correlation in shams). Anatomic data also revealed significant relationships between the volumes of different brain areas within both $\mathrm{HI}$ and shams, but anatomic measures did not correlate with any behavioral measure in the $\mathrm{HI}$ group (though we saw a hippocampal/spatial correlation in shams, in the expected direction). Overall, current data provide an impetus to enhance tools for characterizing individual HI-related pathology in neonates, which could provide more accurate individual prognoses within specific cognitive/behavioral domains and thus improved patient-specific early interventions.

(c) 2015 S. Karger AG, Basel

\section{KARGER 125}

2015 S. Karger AG Basel

0378-5866/15/0375-0440\$39.50/0 


\section{Introduction}

Premature infants ( $<37$ weeks gestational age, GA) and infants born at very low birth weight (VLBW; $<1,500 \mathrm{~g}$ ) are at risk for hypoxic-ischemic (HI) brain injury (lack of blood and/or oxygen) $[1,2]$. The fragility of underdeveloped neurovascular systems (particularly $<32-34$ GA) can lead to intracranial bleeding (e.g. intraventricular/periventricular hemorrhage) [3-5] and ischemic events (e.g. reperfusion failure) [3, 6-8]. Both injuries can cause white matter damage (e.g. periventricular leukomalacia), reflecting the susceptibility of preoligodendrocytes to oxidative stress in the mid-tolast trimester $[9,10]$, with an elevated risk in the extremely premature infant. $\mathrm{HI}$ can also occur in the late preterm infant (34-37 GA), but it manifests here more often as gray matter injury since these regions are undergoing rapid development from 34 weeks to term [11].

Preterm infants with $\mathrm{HI}$ are also at risk for developmental disorders, including delayed cognitive processes [12-14], speech/language delays [13-17], lower IQ scores $[14,18,19]$, spastic cerebral palsy, and memory deficits associated with impaired executive function [20-22]. Though clinicians can speculate about specific developmental impairments that might be expected for individual patients, it remains challenging to predict patient-specific patterns of outcome from the general indices that have been developed to capture the variable and sometimes diffuse nature of neonatal HI-related neuropathology.

One commonly reported deficit following $\mathrm{HI}$ involves impairments in auditory processing, which have been suggested to contribute to language disabilities. Specifically, deficits in rapid auditory processing (RAP) are seen in populations with or at risk for HI (e.g. premature infants) [23-25], and these in turn predict language problems - both in children with $\mathrm{HI}$ insult and other at-risk infants $[15,17,26,27]$. This relationship is thought to reflect the fact that discrimination of rapidly changing sound stimuli, speech or nonspeech stimuli (within tens of milliseconds) is critical to emergent speech perception/ production [15, 28, 29].

Memory impairments are also seen in children with $\mathrm{HI}$, and these can be comorbid with auditory speech/language deficits. Research has shown that VLBW children display lower global and performance IQ coupled with impairments in verbal fluency and expressive/receptive language at $1-7$ years $[30,31]$. Working memory deficits are also associated with language impairments, consistent

Effects of Neonatal HI on Auditory

Processing and Spatial Working Memory with the important role of phonological working memory in language [32]. This comorbidity of HI-related impairments across a broad range of tasks and domains presents a particular impediment to the study of more delineated neurobehavioral associations.

Fortunately, animal models can be used to model HI injury and subsequent behavioral impairments. One well-established rodent HI model (Rice-Vannucci) entails injury induced on postnatal day (P) 7 [33-35]. Although originally thought to simulate term HI (38-40 GA), this model has more recently been thought to simulate late preterm injury, consistent with tissue loss primarily in gray matter and variable amounts of white matter damage [36-39]. Notably, this model produces unilateral injury (via cauterization of the right common carotid artery), so the comparison to human pathology (which typically shows bilateral extent) must be qualified. Nonetheless, the P7 HI rat model provides a useful tool to study the associated functional/behavioral deficits that follow neonatal HI. In fact, studies of P7 HI in rodents show behavioral deficits analogous to those observed clinically, validating long-term neurobehavioral applications. P7 HI male rats show deficits on an RAP task in both prepubertal and adult periods, indicating that deficits persist over time [40-43]. Similarly, deficits on spatial and working memory tasks have been reported in P7 HI rats on a Morris water maze, as well as a more difficult delayed match-to-place radial maze (used to assess spatial learning and working memory, respectively) $[30,42-44]$. The current study sought to explore and replicate well-studied deficits in spatial working memory and RAP in male rats subjected to $\mathrm{HI}$ injury on P7. In addition, the study was designed to assess the following: (1) within-task correlations between juvenile and adult RAP ability and (2) between-task correlations for juvenile RAP and working memory (as assessed in adulthood). Analyses also examined correlations across histological and behavioral measures (shams and HI separately). To our knowledge, such long-term outcome correlations have not been assessed in any model of neonatal HI injury. We hypothesized a replication of deficits in RAP and spatial working memory deficits in P7 HI rats and also that early auditory processing scores would predict subsequent auditory outcomes (in adulthood) particularly in HI-injured subjects. Finally, we sought to determine whether early scores in one domain could predict subsequent outcomes across domains (particularly in HI subjects), consistent with a 'general' injury model where subjects performing poorly on one task also perform poorly on the other. 


\section{Methods}

\section{Subjects}

Time-mated female Wistar rats were delivered to the University of Connecticut vivarium from Charles River (Wilmington, Mass., USA) on embryonic day 7. Dams were housed in tubs (12/12 cycle, food/water ad libitum), with birth on embryonic day 22. Litters were culled ( 8 males, 2 females) on P1. Male animals were used due to the more severe behavioral deficits reported in HI male rats [30, 40, 45], but females were retained until weaning to normalize maternal behavior. Pups were weaned on P21 and pair-housed with like-treated littermates. At P49, subjects were single-housed for further testing.

\section{Induction of $\mathrm{HI}$}

Subjects $(\mathrm{n}=48)$ were assigned to HI $(24)$ or sham (24) within the litter. On P7, male HI animals were anesthetized (isoflurane, $2.5 \%$ ), and an incision was made in the neck. The right common carotid artery was located and cauterized, the incision was sutured, and pups were placed in a temperature-controlled incubator to recover [for further details, see 30,45]. Sham animals were treated similarly but without carotid cauterization. All pups were ID'ed by footpad injection and returned to dams to feed $(2 \mathrm{~h})$. Next, HI pups were placed in an airtight container on a temperature-controlled slide warmer and exposed to $8 \%$ oxygen (balance nitrogen, $120 \mathrm{~min}$ ). Sham animals were placed in a similar container with room air $(120 \mathrm{~min})$. Subjects were then returned to dams until weaning (P21).

\section{Behavioral Testing Design}

All subjects $(\mathrm{n}=48)$ were tested on juvenile auditory processing (silent gap, SG, $0-100$ and $0-10 \mathrm{~ms}$ ) to replicate previous findings $[30,40,45,46]$. Following testing, the animals were randomly split into two groups (A, B; 12 sham and $12 \mathrm{HI}$ in each). Group $\mathrm{A}$ ( $\mathrm{n}=$ $24)$ received additional adult auditory testing, and group $B(n=23$; 1 animal lost due to seizures) was assessed on an 8 -arm radial water maze task.

\section{Auditory Startle Reduction}

The startle reduction paradigm utilizes a subject's acoustic startle reflex - a large motor response to a startle-eliciting stimulus (SES). When the SES is preceded by a prestimulus cue, the acoustic startle reflex is reduced, indicating detection of the cue (also known as prepulse inhibition, PPI). During testing, each animal was placed on a load cell platform (PHM 252B; Med Associates) in a black polypropylene cage in a quiet room. Output voltages were amplified (PHM 250-60), acquired (Biopac MP100A-Ce Acquisition system) and displayed as waveforms (AcqKnowledge). Peak values from the 150-ms epoch after the SES served as the subject's response for a given trial. Auditory stimuli were generated via Pentium III Dell PC and Tucker Davis Technologies (RP2) real-time processor and were amplified (Niles SI-1260 Systems Integration) and delivered via Cambridge Soudworks MC100 loudspeakers. Attenuation (ATT) scores were calculated by average cued-trial peak divided by average uncued-trial peak, multiplied by 100 (cued trials/uncued trials $\times 100$ ) [for further details, see 47]. All acoustic tasks used a 50-ms $105-\mathrm{dB}$ white noise burst as SES, with randomized cue presentation and intertrial intervals $(16,18,22$, or $24 \mathrm{~s})$.
Normal Single Tone: All Animals

The normal single tone (NST; P29, n = 48) task consisted of 103 trials ( 1 session/day). For each trial, a $75-\mathrm{dB}, 7-\mathrm{ms}, 2,300-\mathrm{Hz}$ prepulse cue was presented $50 \mathrm{~ms}$ before the SES (50-ms 105-dB burst; cued trial) or was absent (uncued trial). ATT scores acted as a measure of cue detectability, with scores closer to $100 \%$ indicating poorer performance. This task provided a baseline measure to reveal any hearing or PPI deficits that could affect further testing.

\section{SG $0-100$}

The SG detection task (P33-37 for all animals, $\mathrm{n}=48$; $\mathrm{P} 61$ for group $A, n=24$ ) consisted of 299 trials using SG embedded in 75$\mathrm{dB}$ broadband white noise as a cue. All animals were tested in the juvenile period ( 1 session/day for 5 consecutive days) and group A animals were tested for 1 day in adulthood. On uncued trials, the SES was presented randomly within the broadband white noise (variable intertrial interval). On cued trials, an SG (ranging from $0-100 \mathrm{~ms}$ ) was presented $50 \mathrm{~ms}$ before the SES.

\section{SG $0-10$}

This SG detection task (P40-44 for all animals, $\mathrm{n}=48$; $\mathrm{P} 62-65$ for group $A, n=24$ ) was the same as above, with subjects tested 1 session/day for 5 days in the juvenile period and group A subjects tested for 4 days in adulthood. SG of $0-10 \mathrm{~ms}$ (instead of $0-100 \mathrm{~ms}$ ) served as the cue.

\section{Eight-Arm Radial Water Maze: Group B}

Group B subjects were tested on the maze task (P47-128, n = 23). For a detailed description of the delayed match-to-sample 8 -arm radial water maze, see Chrobak et al. [48]. In brief, the animals were required to locate and mount a submerged platform located in an 8-arm radial water maze. First, the animals were assessed on an acclimation trial to confirm baseline swimming ability, navigation and ability to mount the escape platform. On a test day, the animals received a forced sample trial where only the start and the goal arm were open, 'forcing' the animal to locate the goal platform. After reaching the platform, the animals received a 10 min delay and then a test trial. Here, the animal was placed in a different start arm, with the goal arm remaining the same and all arms open. Testing consisted of 4 such sessions a week for 12 weeks. The goal location and start arm changed daily (sequences of start arms/ goal locations varied systematically among 48 patterns), requiring the animal to keep a representation of the new goal arm in working memory. We recorded the number of errors for each trial, as well as latency per arm choice. A complete analysis of outcome data from this task is reported elsewhere [44]. Finally, 1 day a week, the rats were tested on control trials. During these trials, no forced sample was given, such that the animals had to seek and find the platform without being shown the location, thus providing a measure of chance level performance and swimming ability.

\section{Histological Analysis}

Following completion of testing (P147), the animals were weighed and anesthetized with ketamine/xylazine $(100 \mathrm{mg} / \mathrm{kg} / 15$ $\mathrm{mg} / \mathrm{kg}$ ) and transcardially perfused with $0.9 \%$ saline and $10 \%$ buffered formalin phosphate. Brains were removed, weighed and placed in formalin. Before slicing, brains were placed in a $30 \%$ sucrose solution for cryoprotection, frozen, and then sliced at $60 \mu \mathrm{m}$ using a cryostat. Every third section was mounted and stained (cresyl violet), and every other mounted section was used for analysis. 
Using Stereo Investigator MicroBrightField software on an Axio 2 Zeiss microscope, volumes of the lateral ventricles, hippocampus and cortex were assessed. The fewest sections were counted to achieve a coefficient of error less than 0.05 (stereological validity). Volumes were quantified using $100 \times$ magnification with Cavalieri's Estimator software and a grid overlay. All measurements were performed blind to treatment.

\section{Statistical Analysis}

Statistical analyses were performed using SPSS 19.0 software with an alpha criterion of 0.05 , and two-tailed analyses were used unless otherwise stated.

For auditory scores, a one-way analysis of variance (ANOVA) was initially performed to examine treatment effects on the NST task. A lack of significance confirmed that sham and HI subjects ( $n=24 / 24$ ) had equivalent hearing and PPI. We also assessed cue detection within each group (cued vs. uncued) to confirm detection (paired samples t tests). Further repeated measures ANOVAs were performed on mean ATT scores for SG tasks in juveniles (all animals) and adults (group A) to assess treatment effects (see Results). Notably, to confirm equivalency of group assignment, we analyzed auditory scores obtained from all subjects in the juvenile period only (NST, SG $0-100$, SG $0-10$ ) as a function of group/ treatment. These analyses confirmed the equivalency of the $\mathrm{A} / \mathrm{B}$ sham and A/B HI subgroups (repeated measures ANOVA, Tukey tests), thus confirming that we did not preferentially select poorly performing animals for one subgroup or another.

For the 8-arm radial water maze task, errors per trial across days within 12 testing weeks were averaged into 6 blocks, with scores for each block representing mean errors for a 2 -week test period (2 weeks $=1$ block). A repeated measures ANOVA was performed on average errors made for group B HI and sham animals. Additionally, average latency per choice for each test trial was calculated as total latency to goal divided by errors + 1 (goal arm), and this measure was analyzed by block (repeated measures ANOVA; see Results). Control trial data were also analyzed to confirm equivalent swimming ability, and no treatment effects were seen in either block, confirming that subsequent $\mathrm{HI}$ deficits did not reflect impaired swimming.

Bivariate correlations (Pearson's $r$ ) were also performed using previously determined factors extracted from a varimax rotation factor analysis of behavioral measures. Six factors were extracted and correlated as follows: short auditory gaps, long auditory gaps, errors in 1st half of maze task, errors in 2nd half of maze task, latency per choice in 1st half of maze task, and latency per choice in 2nd half of maze task. These factors were correlated in HI and sham animals separately within each group (see Results).

Finally, to assess neuropathology, volumetric measures of right and left hippocampus, cortex and ventricles were analyzed via individual repeated measures ANOVAs $(\mathrm{HI}=22$, sham $=22)-2 \mathrm{HI}$ and 2 sham animals were excluded from these analyses due to compromised tissue. To assess correlations between anatomy and behavior for HI animals, we calculated a damage ratio score for each brain area (cortex, hippocampus and ventricles) as follows: right volume divided by left volume $(\mathrm{R} / \mathrm{L}) \times 100$. Using these asymmetry measures, we performed additional bivariate correlations with the behavioral factors listed above (short auditory gaps, long auditory gaps, errors in 1st half of maze task, errors in 2nd half of maze task, latency per choice in 1st half of maze task, and latency per choice in 2 nd half of maze task) to indicate whether damage in one brain area was correlated to task performance. For shams, we used a to-

Effects of Neonatal HI on Auditory

Processing and Spatial Working Memory tal volume measure (right volume + left volume) for each brain area, and correlated these values with the same behavioral factors as $\mathrm{HI}$ animals (since damage indices were not relevant to shams).

\section{Results}

\section{Normal Single Tone: All Animals}

Both groups exhibited significant detection of the NST (P30, all animals), as shown by paired samples tests (HI: $\mathrm{t}_{23}=6.232, \mathrm{p}<0.001$; sham: $\left.\mathrm{t}_{23}=3.741, \mathrm{p}<0.002\right)$, with ATT scores significantly lower on cued trials for both groups of animals. A one-way ANOVA for treatment revealed no significant differences $\left(F_{1,47}=2.129, p>0.05\right.$; data not shown), confirming all animals showed PPI and normal hearing. Therefore, any differences in subsequent auditory tasks could be assumed to reflect deficits in processing specific stimuli.

\section{SG 0-100 and SG 0-10: Juvenile}

A 5 (day) $\times 9$ (gap) $\times 2$ (treatment; HI and sham) repeated measures ANOVA assessing juvenile ATT scores on SG 0-100 (P33-37, all animals; fig. 1a, b) revealed a significant overall treatment effect, with $\mathrm{HI}$ animals performing worse than shams $\left(\mathrm{F}_{1,44}=7.252, \mathrm{p}<0.05\right.$; fig. 1a). We also found a significant gap $\times$ treatment effect, indicating that $\mathrm{HI}$ animals performed worse than shams on specific gaps (all subjects unable to detect 2 $\left.\mathrm{ms} ; \mathrm{F}_{8,44}=2.108, \mathrm{p}<0.05\right)$.

A 5 (day) $\times 9$ (gap) $\times 2$ (treatment; $\mathrm{HI}$ and sham $)$ repeated measures ANOVA assessing juvenile ATT scores on SG 0-10 also revealed a significant overall treatment effect, with HI animals performing worse than shams $\left(F_{1,46}=9.595, \mathrm{p}<0.005\right.$; fig. $\left.1 \mathrm{~b}\right)$. We also found a significant gap $\times$ treatment effect, indicating that HI animals performed worse than shams on specific gaps (again with poor performance by all subjects on gaps of $2-3 \mathrm{~ms}$; $\left.\mathrm{F}_{8,46}=5.114, \mathrm{p}<0.001\right)$.

\section{SG 0-100 and SG 0-10: Adulthood}

For group A animals, a 9 (gap) $\times 2$ (treatment; $\mathrm{HI}$ and sham) repeated measures ANOVA (P61-65) revealed a significant overall treatment effect, with $\mathrm{HI}$ animals performing worse than shams on SG $0-100\left(\mathrm{~F}_{1,22}=4.458\right.$, $\mathrm{p}<0.05$; fig. 2a). We also found a significant gap $\times$ treatment effect, indicating that $\mathrm{HI}$ animals performed worse than shams on specific gaps (no detection of $2 \mathrm{~ms} ; \mathrm{F}_{8,22}=$ 2.146, $\mathrm{p}<0.05$ ).

Additionally, a 5 (day) $\times 9$ (gap) $\times 2$ (treatment; HI and sham) repeated measures ANOVA revealed a sig- 

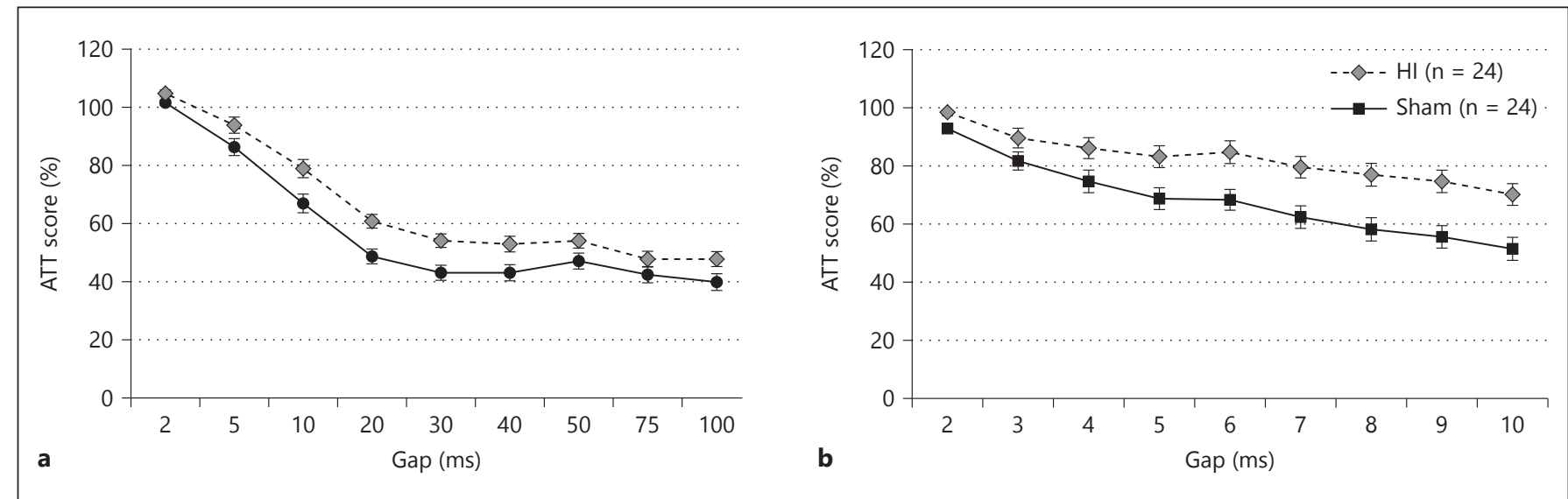

Fig. 1. SG 0-100 and SG 0-10 (juvenile, all animals), a A 5 (day) $\times$ 9 (gap) $\times 2$ (treatment; $\mathrm{HI}$ and sham) repeated measures ANOVA revealed a significant overall treatment effect, with $\mathrm{HI}$ animals performing worse on an SG $0-100$ task (P33-37, p $<0.05)$.

b A 5 (day) $\times 9$ (gap) $\times 2$ (treatment; HI and sham) repeated measures ANOVA revealed a significant overall treatment effect, with $\mathrm{HI}$ animals performing worse on an SG 0-10 task (P40-44, p < $0.05)$.
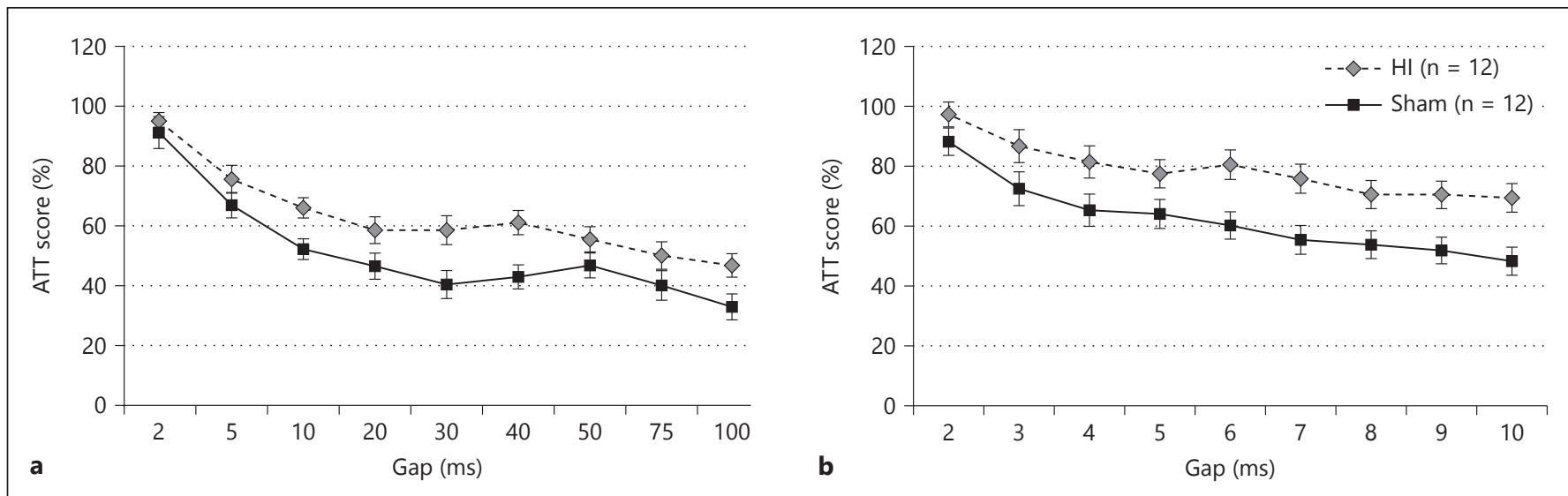

Fig. 2. SG 0-100 and SG 0-10 (adult, group A). a A 9 (gap) $\times 2$ (treatment; HI and sham) repeated measures ANOVA revealed a significant overall treatment effect, with $\mathrm{HI}$ animals performing worse on an SG 0-100 task during adulthood (P61, p < 0.05).

b A 5 (day) $\times 9$ (gap) $\times 2$ (treatment; HI and sham) repeated measures ANOVA revealed a significant overall treatment effect with $\mathrm{HI}$ animals performing worse on an SG 0-10 task during adulthood (P62-65, p < 0.05).

nificant overall treatment effect, with $\mathrm{HI}$ animals performing worse than shams on the SG $0-10$ task $\left(\mathrm{F}_{1,22}=\right.$ $6.969, \mathrm{p}<0.05$; fig. $2 \mathrm{~b}$ ). Again, we found a significant gap $\times$ treatment effect, indicating that $\mathrm{HI}$ animals performed worse than shams on specific gaps $\left(\mathrm{F}_{8,22}=2.174\right.$, $\mathrm{p}<0.05)$.

\section{Correlation Results: Group A}

A bivariate correlation to assess performance on SG tasks in juveniles versus adults for sham animals did not reveal any significant correlations for within-subject per-

formance across age. However, within the juvenile period, performance on long- and short-duration SG was positively correlated $(\mathrm{p}<0.05)$. In adulthood, performance on long- and short-duration SG was again positively correlated $(\mathrm{p}<0.005)$. A similar analysis for $\mathrm{HI}$ animals, on the other hand, revealed a significant positive correlation between performance on long-duration SG in the juvenile period and performance on long-duration $S G$ in adulthood $(p=0.05)$. Additionally, performance on long- and short-duration SG were correlated in the juvenile period $(\mathrm{p}<0.005)$ and in adulthood ( $\mathrm{p}$ 


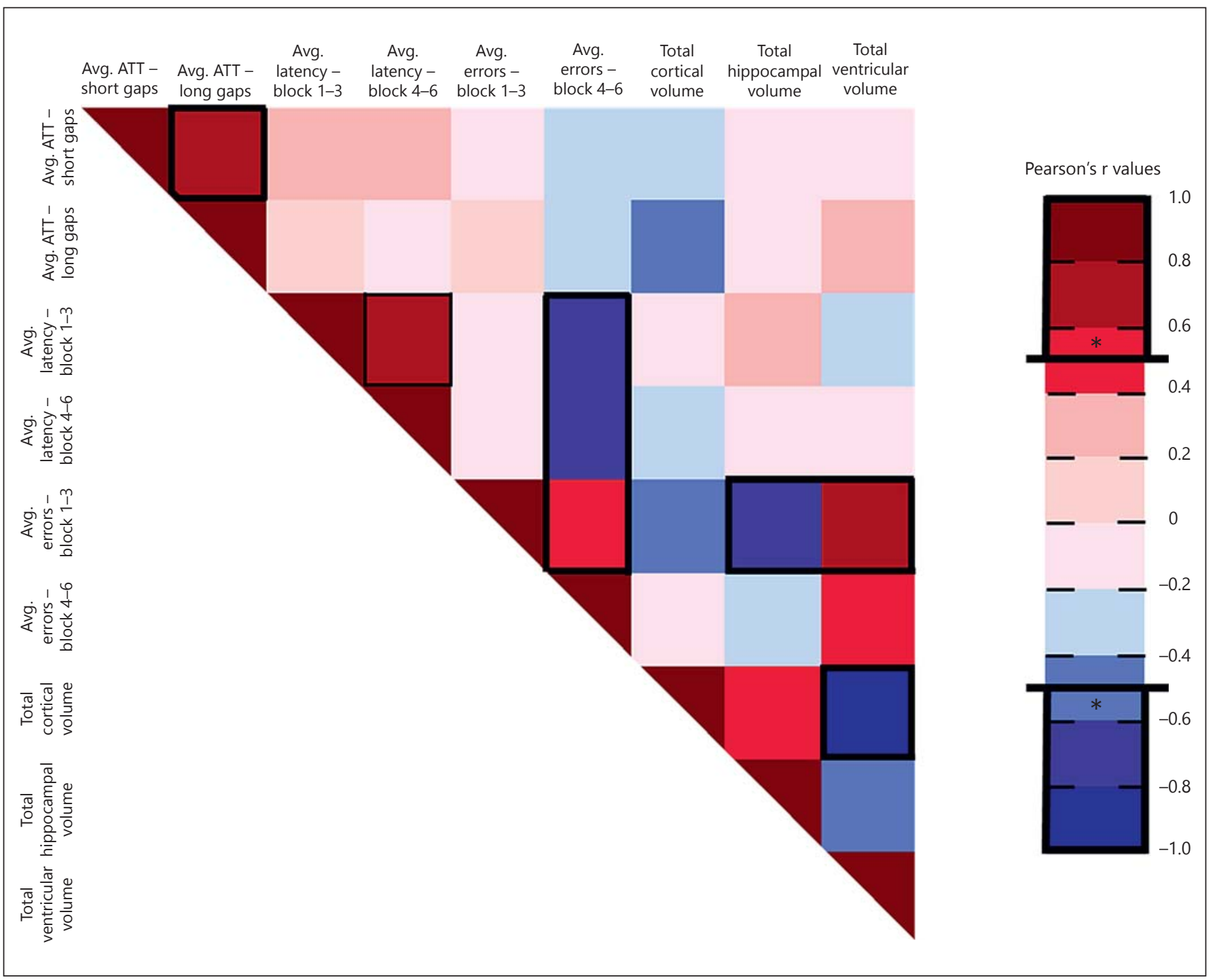

Fig. 3. Correlation matrix for sham animals using 9 factors. Avg. = Average. A bivariate correlation (Pearson's $r$ ) for sham animals revealed significant positive correlations between performance on short- and long-duration SG $(\mathrm{p}<0.05)$. Significant positive correlations were also seen between the 1 st and 2 nd half of maze testing with regard to errors made $(\mathrm{p}<0.05)$. Latency per choice analyses showed a trend to be significantly positively correlated during the 1 st and 2 nd half of maze testing $(p=0.065)$. Significant nega-

0.005) in HI animals. These results are not shown but parallel identical within-task correlations observed in group B (fig. 3, 4).

\section{Eight-Arm Radial Water Maze Task: Group B}

Latencies to mount a visible platform in a water escape task as well as control trials throughout testing revealed no differences between $\mathrm{HI}$ and sham groups, indicating tive correlations were also seen between latency per choice analyses on the 1st half of maze testing and errors during the 2 nd half of maze testing $(\mathrm{p}=0.075)$, as well as latency per choice analyses on the 2 nd half of maze testing and errors during the 2 nd half of testing $(\mathrm{p}<0.05)$. Percent hippocampal damage was positively correlated with errors on both the 1st $(\mathrm{p}<0.05)$ and 2 nd $(\mathrm{p}<0.05)$ half of the maze. Percent ventricular damage correlated negatively with errors in only the 1 st half of the maze $(\mathrm{p}<0.05)$.

that all animals (regardless of $\mathrm{HI}$ injury) could swim. An in-depth evaluation of analyses on submeasures of maze performance as a function of treatment is reported elsewhere [for details, see 44, Study 2]. In brief, a 6 (block) $\times$ 2 (treatment) repeated measures ANOVA revealed a significant overall treatment effect for average errors $\left(\mathrm{F}_{1,21}=8.028, \mathrm{p}<0.05\right)$, with HI performing worse. An additional 6 (block) $\times 2$ (treatment) repeated measures 


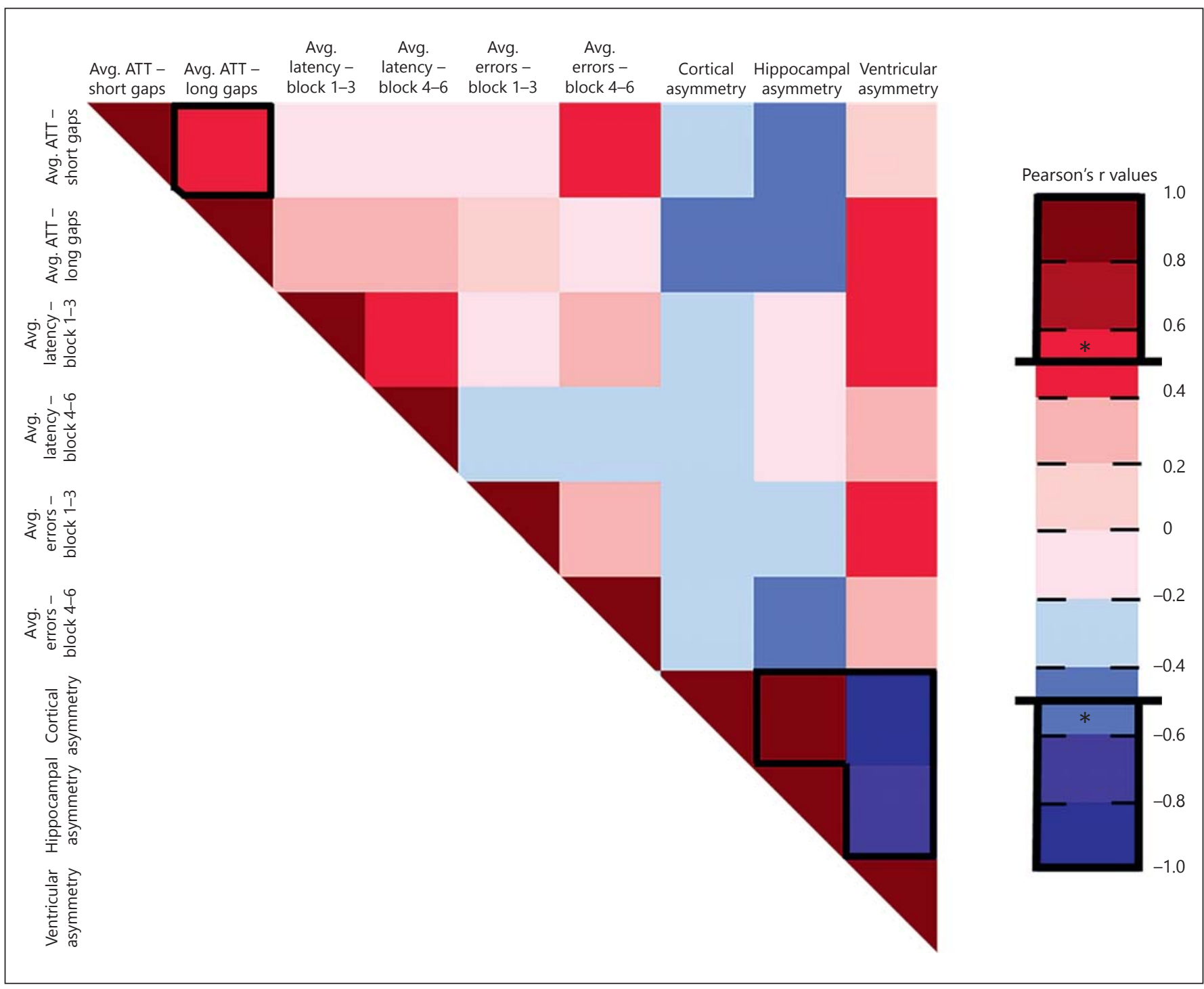

Fig. 4. Correlation matrix for $\mathrm{HI}$ animals using 9 factors. Avg. = Average. A bivariate correlation (Pearson's r) for HI animals revealed a trend for a significant correlation between performance on short- and long-duration SG $(\mathrm{p}<0.07)$. Cortical and hippocampal damage was positively correlated $(\mathrm{p}<0.05)$, whereas cortical and ventricular damage was significantly negatively correlated $(\mathrm{p}<0.05)$. Hippocampal damage and ventricular damage were also significantly negatively correlated $(\mathrm{p}<0.05)$. All other comparisons were not significant.
ANOVA assessing latency per choice also revealed a significant overall treatment effect $\left(\mathrm{F}_{1,21}=7.181, \mathrm{p}<0.05\right)$, indicating that $\mathrm{HI}$ animals took less time to make an arm choice compared to shams [for discussion, see 44] - a possible reflection of impulsivity.

\section{Correlation Results: Group B}

A bivariate correlation to assess juvenile performance across auditory tasks in group B sham animals also revealed significant positive correlations $(\mathrm{p}<0.05$, as seen in group A; fig. 3, 4). There were no significant withinsubject correlations between juvenile auditory scores and adult maze indices for any measure examined. Errors made in the 1st and 2 nd half of maze testing were also significantly positively correlated, while 1 st and 2 nd half latency per choice analyses approached significance $(\mathrm{p}<$ 0.05 and $\mathrm{p}=0.065$, respectively, shams only). Additionally, both 1 st and 2 nd half latency per choice analyses significantly correlated negatively with errors during the 2 nd half of maze testing (longer latencies on both the 1st 


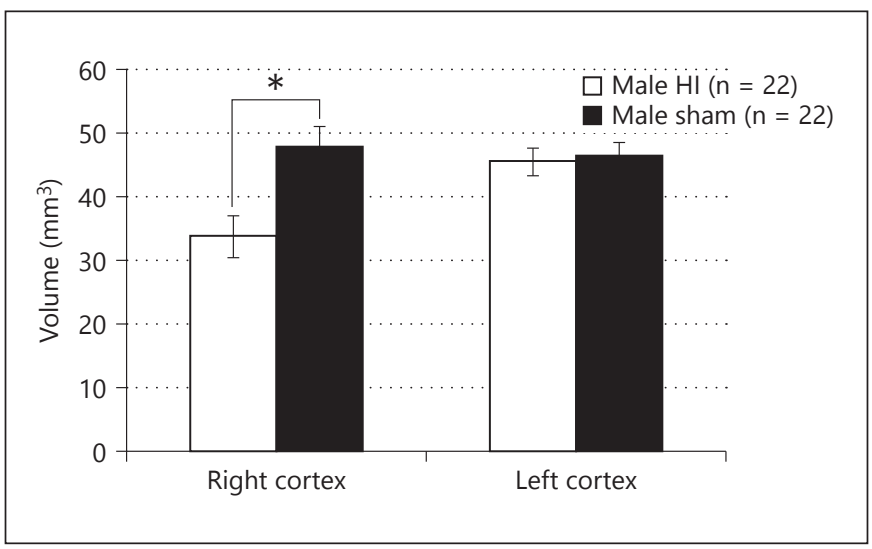

Fig. 5. Right and left cortical volume for male HI and sham rats. An overall 2 (hemisphere) $\times 2$ (treatment) repeated measures ANOVA revealed a significant treatment effect, where $\mathrm{HI}$ animals had significantly smaller overall cortical volume compared to shams $(\mathrm{p}<0.05)$. We also found significant hemisphere $(\mathrm{p}<0.05)$ and hemisphere $\times$ treatment $(\mathrm{p}<0.005)$ effects, indicating that $\mathrm{HI}$ animals had smaller right cortical volumes than shams. ${ }^{*} \mathrm{p}<0.05$.

and 2 nd half of testing associated with fewer errors on the 2nd half of testing; $\mathrm{p}=0.075$ and $\mathrm{p}<0.05$, respectively). This pattern was not seen when examining errors in the 1 st half of maze testing (1st and 2nd half latency per choice performance was not correlated with errors in the 1 st half of maze testing). Taken together, we interpret that sham animals that took longer to make an arm choice were more accurate in maze performance. For HI animals, a similar analysis revealed a trend for significant positive correlations between performance on long- versus short-duration SG in the juvenile period $(\mathrm{p}=0.07)$. No significant correlations (positive or negative) were found between performance on auditory tasks and the maze task (neither performance on long- or short-duration SG correlated with latency or error measures on the maze). In addition, within the maze task, 1 st and 2 nd half latencies were not correlated, nor were latency and/or error measures correlated within or across testing blocks (1-3 vs. $4-6)$.

\section{Histological Results}

An overall 2 (hemisphere) $\times 2$ (treatment) repeated measures ANOVA to assess right and left cortical volume in $\mathrm{HI}$ and sham animals revealed a significant treatment effect, with $\mathrm{HI}$ animals having significantly smaller corti$\mathrm{cal}$ volumes compared to shams $\left(\mathrm{F}_{1,42}=4.615, \mathrm{p}<0.05\right.$; fig. 5). We also found a significant hemisphere $\left(F_{1,42}=\right.$ $8.172, \mathrm{p}<0.05)$ and hemisphere $\times$ treatment $\left(\mathrm{F}_{1,42}=\right.$ 13.437, $\mathrm{p}<0.005)$ effect, indicating that $\mathrm{HI}$ animals had

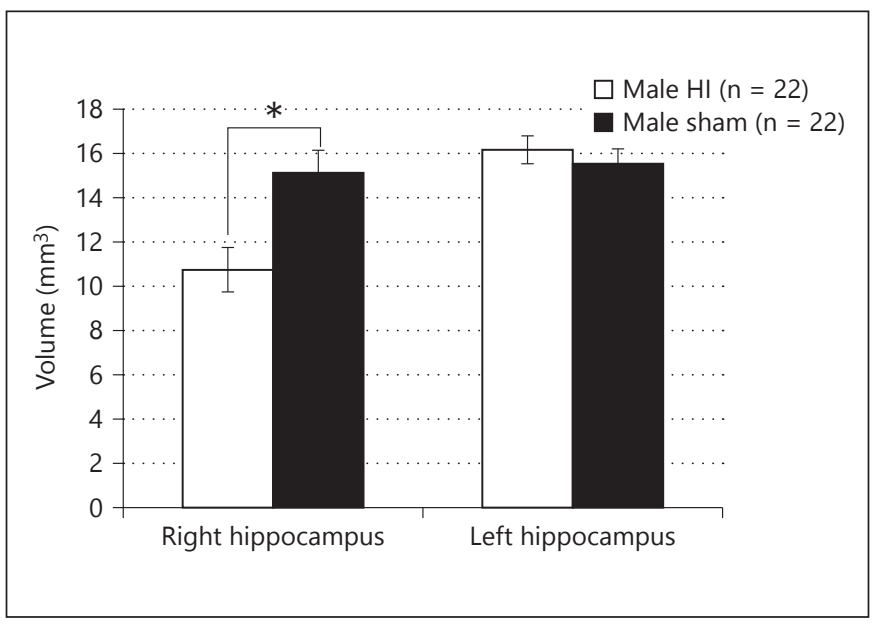

Fig. 6. Right and left hippocampal volume for male HI and sham rats. A 2 (hemisphere) $\times 2$ (treatment) repeated measures ANOVA revealed a significant overall treatment effect $(\mathrm{p}<0.05)$, as well as significant hemisphere $(\mathrm{p}<0.005)$ and hemisphere $\times$ treatment effect $(\mathrm{p}<0.005) .{ }^{*} \mathrm{p}<0.005$.

smaller right cortical volumes than shams. A similar effect was seen when examining hippocampal volume, where a 2 (hemisphere) $\times 2$ (treatment) repeated measures ANOVA revealed a significant overall treatment effect $\left(\mathrm{F}_{1,42}=4.491, \mathrm{p}<0.05\right)$, as well as a significant hemisphere $\left(\mathrm{F}_{1,42}=13.594, \mathrm{p}<0.005\right)$ and hemisphere $\times$ treatment effect $\left(\mathrm{F}_{1,42}=9.669, \mathrm{p}<0.005\right.$; fig. 6). Finally, a 2 (hemisphere) $\times 2$ (treatment) repeated measures ANOVA of ventricular volume revealed a significant hemisphere $x$ treatment effect $\left(\mathrm{F}_{1,42}=10.261, \mathrm{p}<0.005\right)$, with $\mathrm{HI}$ animals showing significantly larger right ventricular volumes compared to shams (fig. 7).

\section{Histological Correlations}

Finally, a bivariate correlation was performed using the factors extracted from the varimax rotation (short auditory gaps, long auditory gaps, errors in 1 st half of maze task, errors in 2nd half of maze task, latency per choice in 1 st half of maze task, and latency per choice in 2 nd half of maze task) and the damage ratio scores for the cortex, hippocampus and ventricles in HI animals. Since this measure was not meaningful in shams, we used a total (left + right) volume score for each of the 3 structures. For sham animals, total hippocampal volume negatively correlated with errors on the 1 st half of the maze $(\mathrm{p}<0.05)$. Additionally, ventricular volume correlated positively with errors in the 1st half of the maze and negatively correlated with total cortical volume $(\mathrm{p}<0.05$ and $\mathrm{p}<0.005$, respectively; fig. 3 ). Conversely, the correlation analysis 


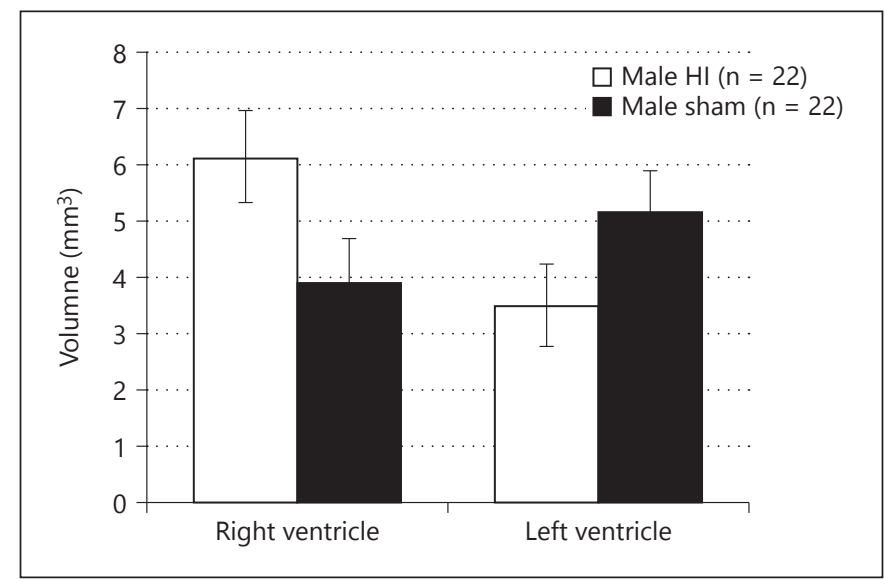

Fig. 7. Right and left ventricular volume for male $\mathrm{HI}$ and sham rats. A 2 (hemisphere) $\times 2$ (treatment) repeated measures ANOVA revealed a significant hemisphere $\times$ treatment effect $(\mathrm{p}<0.005)$, where HI animals had significantly larger right ventricular volumes compared to shams.

for damage indices in $\mathrm{HI}$ animals revealed significant positive correlations between cortical and hippocampal damage $(\mathrm{p}<0.002)$, as well as significant negative correlations between cortical volume and ventricular damage $(\mathrm{p}<0.005)$ and hippocampal and ventricular damage $(\mathrm{p}<$ 0.05 ; fig. 4). No significant correlations were seen between anatomy and behavior in the HI group.

\section{Discussion}

The current study utilized an animal model of P7 HI injury and sought to replicate previously reported rapid auditory processing impairments in male $\mathrm{P} 7$ injured rodents on an SG detection task, as well as to investigate whether early auditory deficits predict auditory deficits in adulthood. In addition, we sought to examine memory impairments on the delayed match-to-position 8-arm radial water maze paradigm and to correlate performance on this task with early auditory processing scores. Here, we hypothesized that performance in the two domains could be related, since auditory and memory impairments typically co-occur following an $\mathrm{HI}$ insult (possibly reflecting a general severity of injury effect across domains) $[32,49]$. We also sought to determine whether damage in specific brain areas correlated with performance on specific behavioral tasks, as might be expected. Results provide interesting and novel evidence in support of two orthogonal neural systems underlying auditory processing and memory.
Replication of Auditory Processing Deficits and Group

\section{A Correlations}

In accord with our hypotheses, we replicated auditory processing deficits in male P7 HI rodents. Specifically, all male HI animals performed significantly worse overall than male shams on both versions of the SG detection task. This significant deficit was apparent on long SG (SG 0-100; fig. 1a) and short SG (SG 0-10; fig. 1b). Moreover, overall significant adult deficits were seen (group A) on SG 0-100 and SG 0-10 (HI subjects again significantly impaired compared to shams; fig. 2a, b). This finding is consistent with prior evidence that behavioral HI deficits persist into adulthood, suggesting a lack of recovery of function in P7 HI animals [40, 42-44, 46]. This is supported by correlations that reveal a significant correlation for performance on long-duration SG in the juvenile and long-duration SG in adulthood in HI animals ( $p=0.05)$. These results are important given clinical research showing that deficits in rapid auditory processing contribute to language deficits as children age $[23,50,51]$.

\section{Deficits on an Eight-Arm Radial Water Maze}

Our hypotheses were also supported with regard to the delayed match-to-position 8-arm radial water maze task. Here, male HI animals made significantly more errors overall (indicative of a memory impairment) compared to shams (group B; fig. 3) [for result details, see 44]. Congruent with auditory results, these findings indicate that behavioral deficits following HI persist into adulthood. Again, the clinical literature is consistent, with substantial evidence pointing to persistent memory deficits in children that undergo an $\mathrm{HI}$ insult [51-54]. In fact, these cognitive deficits may be even more evident as affected children reach school age due to the more complex memory tasks and skills required [55].

\section{Behavioral Correlations between Auditory and Maze} Tasks: Group B

An important aspect of the current study was the ability to assess whether impairments on an auditory task in the juvenile period were correlated with impairments on the match-to-position 8-arm radial maze task in adulthood. Our rationale for exploring this relationship derives from clinical literature indicating that memory and auditory impairments are often comorbid in infants who suffer an HI insult $[49,51,56]$. We examined this issue by examining data from group B subjects, who were tested on both paradigms. Surprisingly, two separate bivariate correlations for $\mathrm{HI}$ and sham animals did not reveal any significant positive or negative correlations for perfor- 
mance on the two different tasks (fig. 3, 4). This finding implies that the underlying mechanisms responsible for auditory processing and spatial memory are orthogonal (deficits or superiority in one area are not predictive of deficits or superiority in another) in both groups of animals. Moreover, it provides insight into our initial question regarding whether HI subjects show domain-specific deficits or cross-domain impairments that are strongly intercorrelated. Our findings suggest that when considering HI damage in the clinical population (where pathology is notably variable), deficits in one behavioral domain may not predict deficits in another behavioral domain $[57,58]$.

Bivariate correlations also revealed that performance on both adult auditory processing tasks (SG 0-100 and SG $0-10$ ) were positively correlated in both $\mathrm{HI}$ and sham animals. This finding supports the presence of a neural subsystem subserving gap detection which functions orthogonally to systems subserving spatial memory. Importantly, the auditory processing data of group A support a within-domain correlation, with long- and short-duration SG performance correlating within and across time points (juvenile and adult). This offsets arguments that maze indices failed to correlate with earlier auditory scores in group B simply because the animals were older and affirms the within-domain stability and reliability of auditory factors.

With regard to internal task stability/validity within our maze task, group B sham animals revealed that both measures within the maze task (1st and 2nd half errors and latency per choice) were correlated, with 1 st and 2 nd half latencies approaching a significant correlation $(\mathrm{p}=$ 0.065 ; fig. 3). Additionally, in the 2 nd half of testing, the number of errors correlated with the 1 st and 2 nd half latency per choice measure. However, unlike auditory factor correlations, both 1st and 2nd half latencies correlated negatively with errors in the 2 nd half of testing. Specifically, longer latencies per choice were associated with fewer errors, suggesting that subjects who took longer to make a choice were more accurate - a finding discussed elsewhere [44]. Taken together, the correlations within and between measures on the maze task also displayed internal validity.

In contrast to sham animals, latency per choice and errors were not correlated for $\mathrm{HI}$ animals, indicating that these two measures are independent of one another and the animals were not taking more time to make more accurate choices. Perhaps a 3rd orthogonal system subserving attention leads to greater accuracy in less impulsive subjects. Given overall shorter latencies among HI sub-
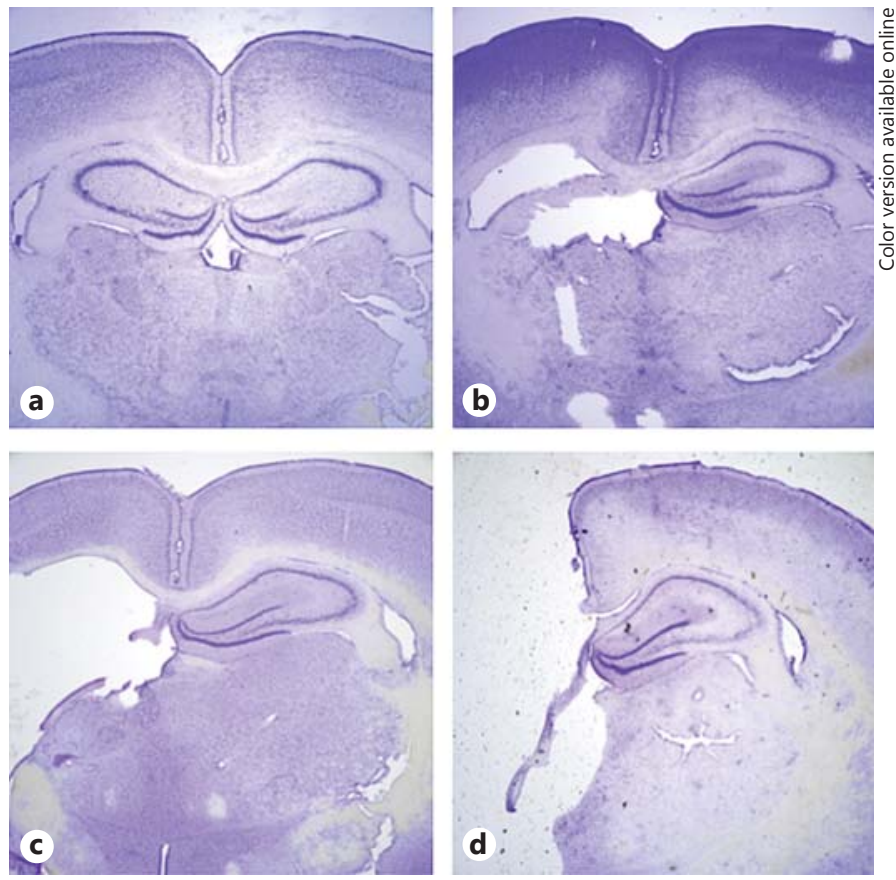

Fig. 8. Representative photomicrographs of Nissl-stained coronal sections representing a typical sham brain (a), mild HI damage (b), moderate HI damage (c), and severe HI damage (d).

jects despite more errors made compared to shams [for discussion, see 44], this attention system could be disrupted in HI subjects and could relate to impulsivity characteristics that have been previously reported in the P7 HI model [44, 59-61]. However, while 'attention' (as indexed by longer latencies per choice) may predict better performance/fewer errors in shams, this association does not appear to be significant on a within-subject basis in the HI group. Possibly, optimal functioning of the attentional system in otherwise healthy (uninjured) rats might predict performance on complex tasks, while damage to this system coupled with comorbid impairments may mask the association within the HI subject group.

\section{Anatomic Results and Anatomic/Behavioral Correlations}

Anatomic results were consistent with prior reports from our laboratory, which showed that the hippocampus, cortex and ventricles of $\mathrm{HI}$ animals were all significantly compromised compared to sham animals [30, 42$44,46]$. Photomicrographs depict the severity of the HI injury in this study, but also indicate substantial variability (fig. 8). Consistent with prior literature, we found that 
HI animals displayed significantly smaller right hippocampal and cortical volumes and larger right ventricular volumes (as shown by a hemisphere $\times$ treatment interaction; fig. 5-7). However, when assessing whether hippocampal, cortical or ventricular volume correlated with auditory and memory impairments, we found no significant correlations in HI animals. For shams, we found a significant negative correlation between hippocampal volume and errors in the first half of the maze. This is consistent with overwhelming evidence that the hippocampus is associated with memory processing [62]. In addition, the significant positive correlation between ventricular volume and errors in the 1st half of the maze, as well as the significant negative correlation between ventricular volume and cortical volume, support the inverse relationship between increased ventricle size and smaller cortical/hippocampal volumes.

These anatomic anomalies are also consistent with clinical reports of significant changes in these brain areas in children born prematurely. For example, studies have shown that VLBW children displayed smaller thalamic and hippocampal volumes than control children and also show anomalies in the dorsolateral prefrontal cortex that could potentially relate to working memory deficits [63]. Other studies point to ventral hippocampal lesions, as well as cortical lesions, as possible factors in later cognitive and behavioral deficits (e.g. memory and auditory processing deficits) $[64,65]$. Future studies should examine more focal brain indices (for example, cellular parameters in auditory structures or connectivity in the prefrontal-striatal pathways), which may ultimately reveal more meaningful correlations between anatomy and behavior in P7 HI animals and also in clinical populations. Given the common use of gross measures of overall neonatal brain damage, the development of more precise and individualized neuropathological profiles could be profoundly useful in the prognosis of individual outcomes and also in the selection of patient-specific interventions.

\section{Conclusion}

The current study successfully replicated previously reported auditory processing deficits in a P7 HI model, as well as memory impairments in a match-to-position 8 -arm radial water maze task. We show that $\mathrm{HI}$ animals exhibited deficits persisting into adulthood, including auditory impairments (group A HI animals) and memory impairments (group B HI animals). In addition, we

found that early auditory processing deficits predict later auditory processing deficits in adulthood (group A HI animals). These combined findings are consistent with clinical reports of similar behavioral deficits in children suffering from $\mathrm{HI}$ insult. Finally, the most interesting finding in the current study is that auditory processing deficits are not correlated with memory deficits in $\mathrm{HI}$ subjects. This was surprising, since these same cross-domain impairments often co-occur in clinical populations. However, our results suggest that HI can differentially affect independent neural systems, and the degree/ pattern of domain-specific injury may be quite variable between subjects (impairments in one cognitive domain might not be predictive of deficits in another cognitive domain). Future research should investigate specific brain regions known to be associated with auditory and memory processes in clinical HI populations and in animal models in order to advance our understanding of specific HI effects on neurobehavioral domains. Such findings would allow clinicians to provide more specific predictions regarding long-term patterns of deficits, along with tailored interventions. Overall, the current results pave the way for further studies to assess brain indices that may be predictive of domain-specific cognitive deficits (rather than a general cross-domain severity of impairment prognosis) following neonatal $\mathrm{HI}$ in the clinical population.

\section{Acknowledgments}

The authors would like to thank all the undergraduates who assisted with behavioral testing. This research was supported by NIH grant HD049792 and internal funding from the University of Connecticut Research Foundation (to R.H.F.).

References 
6 Jensen FE: Developmental factors regulating susceptibility to perinatal brain injury and seizures. Curr Opin Pediatr 2006;18:628-633.

-7 Jantzie LL, Talos DM, Selip DB, An L, Jackson MC, Folkerth RD, Deng W, Jensen FE: Developmental regulation of group 1 metabotropic glutamate receptors in the premature brain and their protective role in a rodent model of periventricular leukomalacia. Neuron Glia Biol 2010;6:277-288.

8 Perlman JM: White matter injury in the preterm infant: an important determination of abnormal neurodevelopment outcome. Early Hum Dev 1998;53:99-120.

-9 Segovia KN, McClure M, Moravec M, Luo NL, Wan Y, Gong X, Riddle A, Craig A, Struve J, Sherman LS, Back SA: Arrested oligodendrocyte lineage maturation in chronic perinatal white matter injury. Ann Neurol 2008;63: 520-523.

10 Haynes RL, Folkerth RD, Keefe RJ, Sung I, Swzeda LI, Rosenberg PA, Volpe JJ, Kinney HC: Nitrosative and oxidative injury to premyelinating oligodendrocytes in periventricular leukomalacia. J Neuropathol Exp Neurol 2003;62:441-450.

-11 Billiards SS, Pierson CR, Haynes RL, Folkerth $\mathrm{RD}$, Kinney HC: Is the late preterm infant more vulnerable to gray matter injury than the term infant? Clin Perinatol 2006;33:915933.

12 van Handel M, Swaab H, de Vries LS, Jongmans MJ: Long-term cognitive and behavioral consequences of neonatal encephalopathy following perinatal asphyxia: a review. Eur J Pediatr 2007;166:645-654.

13 Peterson BS: Brain imaging studies of the anatomical and functional consequences of preterm birth for human brain development. Ann NY Acad Sci 2003;1008:219-237.

14 Steinman KJ, Gorno-Tempini ML, Glidden DV, Kramer JH, Miller SP, Barkovich AJ, Ferriero DM: Neonatal watershed brain injury on magnetic resonance imaging correlates with verbal IQ at 4 years. Pediatrics 2009;123: 1025-1030.

15 Benasich AA, Thomas JJ, Choudhury N, Leppänen $\mathrm{PH}$ : The importance of rapid auditory processing abilities to early language development: evidence from converging methodologies. Dev Psychobiol 2002;40:278-292.

16 Wallace IF, Rose SA, McCarton CM, Kurtzberg D, Vaughan HG Jr: Relations between infant neurobehavioral performance and cognitive outcome in very low birth weight preterm infants. J Dev Behav Pediatr 1995;16: 309-317.

17 Downie AL, Frisk V, Jakobson LS: The impact of periventricular brain injury on reading and spelling abilities in the late elementary and adolescent years. Child Neuropsychol 2005; 11:479-495

18 Aarnoudse-Moens CS, Weisglas-Kuperus N, Duivevoorden HJ, van Goudoever JB, Oosterlaan J: Executive function and IQ predict mathematical and attention problems in very preterm children. PLoS One 2013;8:e55994.
Isaacs EB, Edmonds CJ, Chong WK, Lucas A, Morley R, Gadian DG: Brain morphometry and IQ measurements in preterm children. Brain 2004;127:2595-2607.

20 Luu TM, Ment L, Allan W, Schneider K, Vohr BR: Executive and memory function in adolescents born very preterm. Pediatrics 2011; 127:e639-e646.

21 Burnett AC, Scratch SE, Anderson PJ: Executive function outcome in preterm adolescents. Early Hum Dev 2013;89:215-220.

22 Sun J, Buys N: Early executive function deficit in preterm children and its association with neurodevelopmental disorders in childhood: a literature review. Int J Adolesc Med Health 2012;24:291-299.

23 Mahmoudzadeh M, Dehaene-Lambertz G, Fournier M, Kongolo G, Goudjil S, Dubois J, Grebe R, Wallois F: Syllabic discrimination in premature human infants prior to complete formation of cortical layers. Proc Natl Acad Sci U S A 2013;110:4846-4851.

24 Benasich AA, Tallal P: Infant discrimination of rapid auditory cues predicts later language impairment. Behav Brain Res 2002;136:3149.

25 Benasich AA, Choudhury N, Friedman JT, Realpe-Bonilla T, Chojnowska C, Gou Z: The infant as a prelinguistic model for language learning impairments: predicting from eventrelated potentials to behavior. Neuropsychologia 2006;44:396-411.

26 Jansson-Verkasalo E, Korpilahti P, Jantti V, Valkama M, Vainionpaa L, Alku P, Naatanen R: Neurophysiologic correlates of deficient phonological representations and object naming in prematurely born children. Clin Neurophysiol 2004;115:179-187.

27 Jansson-Verkasalo E, Valkama M, Vainionpaa L, Paakko E, Ilkko E, Lehtihalmes M: Language development in very low birth weight preterm children: a follow-up study. Folia Phoniatr Logop 2004;56:108-119.

28 Fitch RH, Tallal P: Neural mechanisms of language-based learning impairments: Insights from human populations and animal models. Behav Cogn Neurosci Rev 2003;2:155-178.

29 Trehub SE, Henderson JL: Temporal resolution in infancy and subsequent language development. J Speech Hear Res 1996;39:13151320.

30 Smith AL, Alexander M, Rosenkrantz TS, Sadek ML, Fitch RH: Sex differences in behavioral outcome following neonatal hypoxia ischemia: insights from a clinical meta-analysis and a rodent model of induced hypoxic ischemic brain injury. Exp Neurol 2014;254: 54-67.

31 Ortiz-Mantilla S, Choudhury N, Leevers $\mathrm{H}$, Benasich AA: Understanding language and cognitive deficits in very low birth weight children. Dev Psychobiol 2008;50:107-126.

32 McGettigan C, Warren JE, Eisner F, Marshall CR, Shanmugalingam P, Scott SK: Neural correlates of sublexical processing in phonological working memory. J Cogn Neurosci 2011;23:961-977.
33 Rice JE 3rd, Vannucci RC, Brierley JB: The influence of immaturity on hypoxic-ischemic brain damage in the rat. Ann Neurol 1981;9: 131-141.

34 Vannucci RC, Vannucci SJ: A model of perinatal hypoxic-ischemic brain damage. Ann NY Acad Sci 1997;835:234-249.

35 Vannucci RC, Connor JR, Mauger DT, Palmer C, Smith MB, Towfighi J, Vannucci SJ: Rat model of perinatal hypoxic-ischemic brain damage. J Neurosci Res 1999;55:158-163.

36 Patel SD, Pierce L, Ciardiello AJ, Vannucci SJ: Neonatal encephalopathy: pre-clinical studies in neuroprotection. Biochem Soc Trans 2014; 42:564-568.

-37 Fan X, van Bel F, van der Kooij MA, Heijnen CJ, Groenendaal F: Hypothermia and erythropoietin for neuroprotection after neonatal brain damage. Pediatr Res 2013;73:18-23.

38 Fang AY, Gonzalez FF, Sheldon RA, Ferriero DM: Effects of combination therapy using hypothermia and erythropoietin in a rat model of neonatal hypoxia-ischemia. Pediatr Res 2013;73:12-17.

-39 Vannucci RC: Hypoxic-ischemic encephalopathy. Am J Perinatol 2000;17:113-120.

40 Hill CA, Threlkeld SW, Fitch RH: Early testosterone modulated sex differences in behavioral outcome following neonatal hypoxia ischemia in rats. Int J Dev Neurosci 2011a;29: 381-388.

-41 Alexander M, Hill CA, Rosenkrantz TS, Fitch RH: Evaluation of the therapeutic benefit of delayed administration of erythropoietin following early hypoxic-ischemic injury in rodents. Dev Neurosci 2012;34: 515-524.

42 McClure MM, Peiffer AM, Rosen GD, Fitch $\mathrm{RH}$ : Auditory processing deficits in rats with neonatal hypoxic-ischemic injury. Int J Dev Neurosci 2005;4:351-362.

43 McClure MM, Threlkeld SW, Fitch RH: The effects of erythropoietin on auditory processing following neonatal hypoxic-ischemic injury. Brain Res 2006;1087:190-195.

44 Smith AL, Hill CA, Alexander M, Szalkowski CE, Chrobak JJ, Rosenkrantz TS, Fitch RH: Spatial working memory deficits in male rats following neonatal hypoxic ischemic brain injury can be attenuated by task modifications. Brain Sci 2014;4:240-272.

-45 Hill CA, Alexander ML, McCullough LD, Fitch RH: Inhibition of X-linked inhibitor of apoptosis with embelin differentially affects male versus female behavioral outcome following neonatal hypoxia-ischemia in rats. Dev Neurosci 2011b;33:494-504.

46 Alexander M, Garbus H, Smith AL, Rosenkrantz TS, Fitch RH: Behavioral and histological outcomes following neonatal $\mathrm{HI}$ injury in a preterm (P3) and term (P7) rodent model. Behav Brain Res 2014;259;85-96.

-47 Fitch RH, Threlkeld SW, McClure MM, Peiffer AM: Use of a modified prepulse inhibition paradigm to assess complex auditory discrimination in rodents. Brain Res Bull 2008; 76:1-7.
Effects of Neonatal HI on Auditory

Processing and Spatial Working Memory
Dev Neurosci 2015;37:440-452 
48 Chrobak JJ, Hinman JR, Sabolek HR: Revealing past memories: proactive interference and ketamine-induced memory deficits. J Neurosci 2008;28:4512-4520.

-49 Woodward LJ, Moor S, Hood KM, Champion PR, Foster-Cohen S, Inder TE, Audtin NC: Very preterm children show impairments across multiple neurodevelopmental domains by age 4 years. Arch Dis Child Fetal Neonatal Ed 2009;94:F339-F344.

50 Downie ALS, Jakobson LS, Frisk V, Ushycky I: Auditory temporal processing deficits in children with periventricular brain injury. Brain Lang 2002;80:208-225.

51 de Kleine MJ, den Ouden AL, Kollee LA, Nihuis-van der Sanden MW, Sondaar M, van Kessel-Feddema BJ, Knuijt S, van Baar AL, Ilsen A, Breaur-Pieterse R, Briet JM, Brand R, Verloove-Vanhorick SP: Development and evaluation of a follow-up assessment of preterm infants at 5 years of age. Arch Dis Child 2003;88:870-875.

52 Orchinik LJ, Taylor HG, Espy KA, Minich N Klein N, Sheffield T, Hack M: Cognitive outcomes for extremely preterm/extremely low birth weight children in kindergarten. J Int Neuropsychol Soc 2011;17:1067-1079.

53 Potharst ES, van Wassenaer-Leemhuis AG, Houtzager BA, Livesey D, Kok JH, Last BF, Oosterlaan J: Perinatal risk factors for neurocognitive impairments in preschool children born very preterm. Dev Med Child Neurol 2013;55:178-184.
54 Luciana M: Cognitive development in children born preterm: implications for theories of brain plasticity following early injury. Dev Psychopathol 2003;15:1017-1047.

55 Sansavini A, Guarini A, Caselli MC: Preterm birth: neuropsychological profiles and atypical developmental pathways. Dev Disabil Res Rev 2011;17:102-113.

56 Curtis WJ, Zhuang J, Townsend EL, Hu X, Nelson CA: Memory in early adolescents born prematurely: a functional magnetic resonance imaging investigation. Dev Neuropsychol 2006;29:341-377.

57 Barrett RD, Bennet L, Davidson J, Dean JM, George S, Emerald BS, Gunn AJ: Destruction and reconstruction: hypoxia and the developing brain. Birth Defects Res C Embryo Today 2007;81:163-176.

58 Volpe JJ: The encephalopathy of prematurity - brain injury and impaired brain development inextricably intertwined. Semin Pediatr Neurol 2009a;16:167-178.

59 Oorschot DE, Voss L, Covey MV, Bilkey DK, Saunders SE: ADHD-like hyperactivity, with no attention deficit, in adult rats after repeated hypoxia during the equivalent of extreme prematurity. J Neurosci Methods 2007;166: 315-322.
60 Spencer TJ: Attention-deficit/hyperactivity disorder: diagnosis, lifespan, comorbidities, and neurobiology. Ambul Pediatr 2007; 7(suppl):73-81.

61 Biederman J: Growth deficits and attentiondeficit/hyperactivity disorder revisited: Impact of gender, development, and treatment. Pediatrics 2003;111:1010-1016.

62 Beauchamp MH, Thompson DK, Howard K, Doyle LW, Egan GF, Inder TE, Anderson PJ: Preterm infant hippocampal volumes correlate with later working memory deficits. Brain 2008;131:2986-2994.

63 Lowe J, Duvall SW, MacLean PC, Caprihan A, Ohls R, Qualls C, Phillips J: Comparison of structural magnetic resonance imaging and development in toddlers born very low birth weight and full-term. J Child Neurol 2011;26: 586-592.

64 Marquis JP, Goulet S, Dore FY: Neonatal lesions of the ventral hippocampus in rats lead to prefrontal cognitive deficits at two maturational stages. Neuroscience 2006;140:759767.

65 Peterson BS, Vohr B, Staib LH, Cannistraci CJ, Dolberg A, Schneider KC, Katz KH, Westerveld M, Sparrow S, Anderson AW, Duncan CC, Makuch RW, Gore JC, Ment LR: Regional brain volume abnormalities and long-term cognitive outcome in preterm infants. JAMA 2000;284:1939-1947. 